\title{
SKEW CONNECTIONS IN VECTOR BUNDLES AND THEIR PROLONGATIONS
}

\author{
JURAJ VIRSIK \\ (Received 5 January 1970) \\ Communicated by E. Strzelecki
}

The paper is closely related to [1] and [2]. A skew connection in a vector bundle $E$ as defined here is a pseudo-connection (in the sense of [1]) which can be changed into a connection by transforming separately the bundle $E$ itself and the bundle of its differentials, i.e. one-forms on the base with values in $E$. The properties of skew connections are thus expected to be only "algebraically" more complicated than those of connections; especially one can follow the pattern of [1], and prolong them to obtain higher order semi-holonomic and non-holonomic pseudo-connections. It is shown in this paper that under some circumstances the main theorem of [1] or [2] applies also to skew connections.

Let $M$ be a fixed (finite-dimensional, $C^{\infty}$-differentiable) manifold, $E$ a (finitedimensional over the reals, $C^{\infty}$-differentiable) vector bundle with base $M$ and fibre type $R^{n}$. Let the dimension of $M$ be $m$. We shall always suppose that the structure group of a vector bundle is the maximal linear group (i.e. $G L(n, R)$ in the case of $E$ ), and neglect the question of its possible reducibility. Let $F$ be another vector bundle over $M, p: E \rightarrow M$ and $p^{\prime}: F \rightarrow M$ the corresponding projections. A $C^{\infty}$-map $\Phi: E \rightarrow F$ (a diffeomorphism), such that $p^{\prime} \Phi=p$ and $\Phi$ is linear on each fibre, is called a bundle morphism (isomorphism). If $T(M)$ and $T(M)^{*}$ are the tangent and cotangent bundles respectively to $M$, denote $T^{1}(E)=E \oplus E \otimes T(M)^{*}$, and by $S^{1}(E)$ the vector bundle over $M$ of all one-jets of local sections of $E$. Denoting by $R$ the trivial bundle $M \times R$, we have clearly $S^{1}(R)=T^{1}(R)$. Note that the fibres of both $S^{1}(E)$ and $T^{1}(E)$ have the same dimension, and that $E \otimes T(M)^{*}$ can be regarded as a subbundle of both $T^{1}(E)$ and $S^{1}(E)$, identifying it with $\operatorname{Ker} \pi_{T}$ and $\operatorname{Ker} \pi_{S}$ respectively, where $\pi_{T}: T^{1}(E) \rightarrow E$ and $\pi_{S}: S^{1}(E) \rightarrow E$ are the natural bundle projections. In [1] we have defined a pseudo-connection in $E$ as a bundle isomorphism $H: S^{1}(E) \rightarrow T^{1}(E)$, and we have seen that it corresponds to a usual connection iff $\pi_{T} H=\pi_{S}$ and $H$ is the identity on $E \otimes T(M)^{*}$.

Let $H=H_{1}+H_{2}$ be a pseudo-connection, where $H_{1}: S^{1}(E) \rightarrow E$ and $H_{2}: S^{1}(E) \rightarrow E \otimes T(M)^{*}$ are its natural composants. It is called a skew connection iff it preserves the subbundle $E \otimes T(M)^{*}$, i.e. iff $\pi_{S}(X)=0 \Rightarrow H_{1}(X)=0$. We have the evident 
LEMMA 1. A pseudo-connection $H$ is a skew connection iff any of the two conditions is satisfied:

(A) There is a bundle morphism $A: E \rightarrow E$ such that $H_{1}=A \pi_{S}$;

(B) There is a bundle morphism $Q: E \otimes T(M)^{*} \rightarrow E \otimes T(M)^{*}$ such that $\pi_{S}(X)=0$ $\Rightarrow H(X)=H_{2}(X)=Q(X)$.

Note that if such $A$ or $Q$ exists for a pseudo-connection $H$, then both they exist, are uniquely determined and invertible (i.e. bundle isomorphisms). Call $A$ the first and $Q$ the second tensor of the skew connection $H$. A pseudo-connection is thus a connection iff it is a skew connection with trivial (i.e. identity) first and second tensors. A skew connection is called a relative connection with respect to a bundle isomorphism $A: E \rightarrow E$ (or briefly an A-connection) if its first and second tensors are $A$ and $A \otimes i d_{(T) M^{*}}$ respectively.

REMARK. A pseudo-connection is a skew connection, iff its components $\Gamma_{k \beta}^{h \alpha}(h, k=1, \cdots n ; \alpha, \beta=0,1, \cdots m)$ in coordinate neighbourhoods (c.f. [1]) satisfy $\Gamma_{k 0}^{h i}=0(h, k=1, \cdots n ; i=1, \cdots m)$. In this case $\Gamma_{k 0}^{h 0}$ are the components of the first, and $\Gamma_{k j}^{h i}$ the components of the second tensors.

Both the groups Aut $S^{1}(E)$ or Aut $T^{1}(E)$, of all bundle automorphisms of $S^{1}(E)$ or $T^{1}(E)$ respectively, act freely and transitively (to the right or left respectively) on the set $P C(E)$ of all pseudo-connections in $E$. Each element $B \in$ Aut $T^{1}(E)$ is uniquely determined by a 'matrix of tensors' $\left(B_{i k}\right)_{i, k=1,2}$, where $B_{11}$ : $E \rightarrow E, B_{12}: E \rightarrow E \otimes T(M)^{*}, B_{21}: E \otimes T(M)^{*} \rightarrow E, B_{22}: E \otimes T(M)^{*} \rightarrow E \otimes$ $T(M)^{*}$ are bundle morphisms subject only to the condition that the morphism $(X+Y) \mapsto\left(B_{11}(X)+B_{21}(Y)\right)+\left(B_{12}(X)+B_{22}(Y)\right)$ of $T^{1}(E)$ onto itself be invertible.

THEOREM 1. The subset $S C(E) \subset P C(E)$ of skew connections in $E$ is one of the orbits in $P C(E)$ with respect to the action of the subgroup $\mathscr{B} \subset$ Aut $T^{\mathbf{1}}(E)$ characterized by the condition $B_{21}=0$.

The proof is evident. Note that $B_{21}=0$ implies the invertibility of both $B_{11}$ and $B_{22}$.

THEOREM 2. If $H$ is a skew connection in $E$, its first and second tensors being $A$ and $Q$ respectively, and $B \in \mathscr{B}$, then the first and second tensors of the skew connection $B H$ are $B_{11} A$ and $B_{22} Q$ respectively.

The proof is again evident as well as that of the

COROllary. Given any pair $A: E \rightarrow E, Q: E \otimes T(M)^{*} \rightarrow E \otimes T(M)^{*}$ of bundle isomorphisms, there is a unique orbit $C_{A Q}(E) \subset P C(E)$, with respect to the action of the subgroup $\mathscr{B}_{0} \subset \mathscr{B} \subset$ Aut $T^{1}(E)$, consisting of all the skew connections in E admitting $A$ and $Q$ as their first and second tensors. The subgroup $\mathscr{B}_{0}$ is characterized by the condition $B_{21}=0, B_{11}=i d_{E}, B_{22}=i d_{E \otimes T T_{M} *}$. 
If $H$ is a skew connection, $A$ and $Q$ its tensors as above, let $B$ be defined by the quadrupole $B_{11}=A^{-1}, B_{21}=B_{12}=0, B_{22}=Q^{-1}$. Then $H^{0}=B H$ is a connection in $E$ called the associated with $H$ connection. Conversely, if $H^{0}$ is a connection in $E, A, Q$ arbitrary bundle automorphisms as above, then $H=B^{-1} H^{0}$, where $B^{-1}$ is the inverse of $B$ as above, is a skew connection admitting $A$ and $Q$ as its first and second tensors respectively. Explicitly

$$
H=j_{T}^{1} A \pi_{T} H^{0}+j_{T}^{*} Q \pi_{T}^{*} H^{0},
$$

where $T^{1}(E)$ is represented by the direct sum diagram

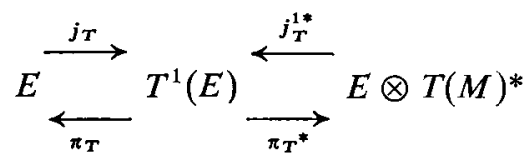

(c.f. [1]). There is hence a natural one-to-one-correspondence between $S C(E)$ and all the triples consisting of connections in $E$ and bundle automorphisms $A: E \rightarrow E, Q: E \otimes T(M)^{*} \rightarrow E \otimes T(M)^{*}$.

Remark. If $B \in$ Aut $T^{1}(E), \quad B^{\prime} \in \mathscr{B}_{0} B$ then $B_{21}^{\prime}=B_{21}, B_{22}^{\prime}=B_{22} ;$ if moreover $B_{21}=0$, then also $B_{11}^{\prime}=B_{11}$. Thus the tensors $B_{21}, B_{22}$ are invariants of the right cosets with respect to $\mathscr{B}_{0}$; i.e. given $H \in P C(E)$, the tensors $B_{21}=B_{21}(H)$ and $B_{22}=B_{22}(H)$ corresponding to any automorphism of $T^{1}(E)$ taking $H$ into a connection are "invariants of the pseudo-connection $H$ ". It is a skew connection iff $B_{21}(H)=0$; in that case also $B_{11}=B_{11}(H)$ is an 'invariant' and evidently $B_{11}(H)^{-1}$ and $B_{22}(H)^{-1}$ coincide with the first and second tensors of the skew connection $H$.

Let $\Phi: E \rightarrow E$ be a bundle morphism. We have then also bundle morphisms $S^{1}(\Phi): S^{1}(E) \rightarrow S^{1}(E)$ and $T^{1}(\Phi): T^{1}(E) \rightarrow T^{1}(E)$ (c.f. [1]); $S^{1}$ and $T^{1}$ are functors from the category of vector bundles over $M$ into itself. A skew connection $H$ in $E$ is called $\Phi$-invariant if $T^{1}(\Phi) H=H S^{1}(\Phi)$. We have again an evident

Lemma 2. If $H \in S C(E)$ is $\Phi$-invariant, then so is any skew connection $B H$, where $B \in \mathscr{B}_{0}$ and $B_{11}$ commutes with $\Phi, B_{22}$ with $\Phi \otimes i d_{T(M)^{*}}$.

CoRollary. A skew connection is $\Phi$-invariant if the associated connection is $\Phi$-invariant and $\Phi$ commutes with the first tensor, $\Phi \otimes i d_{T(M)}$ with the second tensor.

A skew connection is called regular, if it is $A$-invariant, where $A$ is its first tensor. Thus such $H \in S C(E)$ is regular if its associated connection is $A$-invariant and $A \otimes i d_{T(M)^{*}}$ commutes with the second tensor; especially an $A$-connection is regular if its associated connection is $A$-invariant.

Remark. The $\Phi$-invariancy of a connection $H$, i.e. the condition $H S^{1}(\Phi)=$ $T^{1}(\Phi) H$, is equivalent with the condition $\nabla_{X}(\Phi f)=\Phi \nabla_{X} f$ for any local section $X$ of $T(M)$ and any local section $f$ of $E$, where $\nabla_{X} f=\left\langle X, H_{2}\left(j^{1} f\right)\right\rangle$ is the co- 
variant derivative induced by the connection $H$ (c.f. [1], p. 144). In other words $H$ is $\Phi$-invariant iff the absolute differential of $\Phi$ is zero. This gives also the local conditions for the regularity of a skew connection in terms of its components $\Gamma_{k}^{h}$ and $\Gamma_{k i}^{h}$ as

$$
\partial_{i} \Gamma_{k}^{s}+\sum_{h=1}^{n}\left(\Gamma_{h i}^{s} \Gamma_{k}^{h}-\Gamma_{k i}^{h} \Gamma_{h}^{s}\right)=0
$$

for each $i=1, \cdots m ; s, k=1, \cdots n$.

If $E$ and $F$ are two vector bundles over $M, H_{E}$ and $H_{F}$ connections in $E$ and $F$ respectively, then they induce natural connections $H_{E}(\oplus) H_{F}$ in $E \oplus F$ and $H_{E}(\otimes) H_{F}$ in $E \otimes F$; there is also a connection $H_{E^{*}}$ in the dual bundle $E^{*}$ induced by the connection $H_{E}$ (see e.g. again [1] including the notations). Trying to generalize this to arbitrary skew connections $H_{E}$ and $H_{F}$ with the first tensors $A_{E}$ and $A_{F}$, the second tensors $Q_{E}$ and $Q_{F}$ respectively, we first pass to the associated connections $H_{E}^{0}, H_{F}^{0}$, form $H_{E}^{0}(\oplus) H_{F}^{0}$ or $H_{E}^{0}(\otimes) H_{F}^{0}$ or $H_{E^{*}}^{0}$ as above, and introduce $H_{E}(\oplus) H_{F}$ or $H_{E}(\otimes) H_{F}$ or $H_{E^{*}}$ as the skew connections with these associated connections and the tensors 'naturally' connected with those of $H_{E}$ and $H_{F}$. In the case of the direct sum this means that we put $A_{E \oplus F}=A_{E} \oplus A_{F}$, $Q_{E \oplus F}=Q_{E} \oplus Q_{F}$ for the tensors of $H_{E}(\oplus) H_{F}$, but in the case of the tensor product, to obtain the second tensor reasonably linked with $Q_{E}$ and $Q_{F}$, one has to suppose that $Q_{E}=P_{E} \otimes R, Q_{F}=P_{F} \otimes R$, where $P_{E}: E \rightarrow E, P_{F}: F \rightarrow F$, $R: T(M)^{*} \rightarrow T(M)^{*}$ are some bundle automorphisms. We shall refer to this situation by saying that $H_{E}$ and $H_{F}$ are $R$-linked. Now if the skew connections $H_{E}$ and $H_{F}$ are $R$-linked, we define the tensors of $H_{E}(\otimes) H_{F}$ and $H_{E}$ by $A_{E \otimes F}=$ $A_{E} \otimes A_{F}, Q_{E \otimes F}=P_{E} \otimes P_{F} \otimes R$ and $A_{E^{*}}=\left(A_{E}\right)^{*}, Q_{E^{*}}=\left(P_{E}\right)^{*} \otimes R$. Note that if $H_{E}$ is an $A_{E}$-connection, $H_{F}$ an $A_{F}$-connection, then they are linked by the identity and $H_{E}(\otimes) H_{F}$ is an $\left(A_{E} \otimes A_{F}\right)$-connection.

An easy consequence of Lemma 3.1 and 3.2 in [1] is

Lemma 3. If $\Phi: E \rightarrow E, \Psi: F \rightarrow F$ are bundle morphisms, $H_{E}$ and $H_{F}$ connections in $E$ and $F$ respectively, then

and

$$
H_{E} S^{1}(\Phi)(\otimes) H_{F} S^{1}(\Psi)=\left(H_{E}(\otimes) H_{F}\right) S^{1}(\Phi \otimes \Psi)
$$

$$
T^{1}(\Phi) H_{E}(\otimes) T^{1}(\Psi) H_{F}=T^{1}(\Phi \otimes \Psi)\left(H_{E}(\otimes) H_{F}\right) .
$$

Lemma 4. Let $\Phi: E \rightarrow E, \Psi: F \rightarrow F$ be bundle morphisms. Let $H_{E}$ be a $\Phi$ invariant connection in $E, H_{F}$ a $\Psi$-invariant connection in $F$. Then

(a) $H_{E}(\oplus) H_{F}$ is $(\Phi \oplus \Psi)$-invariant,

(b) $H_{E}(\otimes) H_{F}$ is $(\Phi \otimes \Psi)$-invariant,

(c) $H_{E^{*}}$ is $\Phi^{*}$-invariant.

Proof. (a) If $E \oplus F$ is represented by 


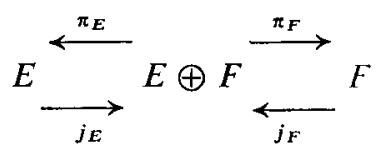

then $H_{E}(\oplus) H_{F}=T^{1}\left(j_{E}\right) H_{E} S^{1}\left(\pi_{E}\right)+T^{1}\left(j_{F}\right) H_{F} S^{1}\left(\pi_{F}\right)$ (c.f. (3.23) in [1]) and hence $H_{E} S^{1}(\Phi)=T^{1}(\Phi) H_{E}, H_{F} S^{1}(\Psi)=T^{1}(\Psi) H_{F}$ implies

$$
\begin{aligned}
\left(H_{E}(\oplus) H_{F}\right) S^{1}(\Phi \oplus \Psi)=T^{1}\left(j_{E}\right) H_{E} S^{1}\left(\Phi \pi_{E}\right)+T^{1}\left(j_{F}\right) H_{F} S^{1}\left(\Psi \pi_{F}\right) \\
=T^{1}\left(j_{E} \Phi\right) H_{E} S^{1}\left(\pi_{E}\right)+T^{1}\left(j_{F} \Psi\right) H_{F} S^{1}\left(\pi_{F}\right)=T^{1}(\Phi \oplus \Psi) T^{1}\left(j_{E}\right) H_{E} S^{1}\left(\pi_{E}\right) \\
\\
\quad+T^{1}(\Phi \oplus \Psi) T^{1}\left(j_{F}\right) H_{F} S^{1}\left(\pi_{F}\right) .
\end{aligned}
$$

(b) follows directly from Lemma 3.3 in [1].

(c) Denoting by $c: E \otimes E^{*} \rightarrow \boldsymbol{R}$ the natural contraction, we have $c\left(i d_{E} \otimes\right.$ $\left.\Phi^{*}\right)=c\left(\Phi \otimes i d_{E^{*}}\right)$ and thus applying this, Lemma 3 and $(b)$ to the relation $T^{1}(c)$ $\left(H_{E}(\otimes) H_{E^{*}}\right)=S^{1}(c)$, (c.f. (3.49) in [1]), we get

$$
\begin{aligned}
T^{1}(c)\left(H_{E}(\otimes)\right. & {\left.\left[H_{E^{*}} S^{1}\left(\Phi^{*}\right)\right]\right)=T^{1}(c)\left(H_{E}(\otimes) H_{E^{*}}\right)\left(S^{1}\left(i d_{E}\right) \otimes S^{1}\left(\Phi^{*}\right)\right) } \\
= & S^{1}(c)\left(S^{1}(\Phi) \otimes S^{1}\left(i d_{E^{*}}\right)\right)=T^{1}(c)\left(\left[H_{E} S^{1}(\Phi)\right](\otimes) H_{E^{*}}\right) \\
= & \left.T^{1}(c)\left(T^{1}(\Phi) H_{E}\right](\otimes) H_{E^{*}}\right)=T^{1}(c) T^{1}\left(i d_{E} \otimes \Phi^{*}\right)\left(H_{E}(\otimes) H_{E^{*}}\right) \\
= & T^{1}(c)\left(H_{E}(\otimes)\left[T^{1}\left(\Phi^{*}\right) H_{E^{*}}\right]\right) .
\end{aligned}
$$

Now according to the uniqueness property in Lemma 3.5 in [1], the proof is completed.

Corollary. Let $H_{E}$ and $H_{F}$ be regular skew connections in $E$ and $F$ respectively, their tensors being $A_{E}$ or $Q_{E}=P_{E} \otimes R$, and $A_{F}$ or $Q_{F}=P_{F} \otimes R$. Let $A_{E}$ commute with $P_{E}$ and $A_{F}$ with $P_{F}$. Then the skew connections $H_{E}(\oplus) H_{F}$, $H_{E}(\otimes) H_{F}$ and $H_{E^{*}}$ are regular.

ProOF. It is sufficient to show that $\left(A_{E} \oplus A_{F}\right) \otimes i d_{T(M)} *$ commutes with $Q_{E} \oplus Q_{F}$, and $A_{E} \otimes A_{F} \otimes i d_{T(M)^{*}}$ with $P_{E} \otimes P_{F} \otimes R$, as well as $\left(A_{E}\right)^{*} \otimes i d_{T(M)^{*}}$ with $\left(P_{E}\right)^{*} \otimes R$; but this is obvious from the assumptions.

This corollary is useful for the prolongation procedure of skew connections. First let us recall briefly some basic notions and notations from [1], (c.f. also [2]).

For each integer $q \geqq 1$ denote by $S^{q}, \bar{S}^{q}$ and $\bar{S}^{q}$ the covariant functors from the category of vector bundles over $M$ into itself which are defined by means of the holonomic, semi-holonomic and non-holonomic jet prolongations respectively in the sense of Ch. Ehresmann. We put $E=S^{0}(E)=\bar{S}^{0}(E)=\tilde{S}^{0}(E)$ as well as $E=T^{0}(E)=\bar{T}^{0}(E)=\tilde{T}^{0}(E)$ and define for each $q \geqq 1$ recurrently

$$
\begin{aligned}
& T^{q}(E)=T^{q-1}(E) \oplus E \otimes\left(\bigcirc T(M)^{*}\right) \\
& T^{q}(E)=\bar{T}^{q-1}(E) \oplus E \otimes\left(\otimes T(M)^{*}\right) \\
& T^{q}(E)=\tilde{T}^{q-1}(E) \oplus \tilde{T}^{q-1}(E) \otimes T(M)^{*},
\end{aligned}
$$


giving rise to the functors $T^{q}, \bar{T}^{q}, \widetilde{T}^{q}$ from the category of vector bundles into itself. Let $\pi_{S}^{q}: S^{q}(E) \rightarrow S^{q-1}(E), \pi_{S}^{q}: \bar{S}^{q}(E) \rightarrow \bar{S}^{q-1}(E)$ and $\pi_{S}=\tilde{\pi}_{S}^{q}: \tilde{S}^{q}(E)=$ $S^{1}\left(\tilde{S}^{q-1}(E)\right) \rightarrow \tilde{S}^{q-1}(E)$, or correspondingly $\pi_{T}^{q}, \bar{\pi}_{T}^{q}$ and $\tilde{\pi}_{T}^{q}$ (c.f. (1)) be the natural surjections. Let further $\imath_{S}^{q}: S^{q}(E) \rightarrow \bar{S}^{q}(E), i_{S}^{q}: \bar{S}^{q}(E) \rightarrow \widetilde{S}^{q}(E)$ denote the natural injections as well as $\imath_{T}^{q}$ and $i_{T}^{q}$ in the other case. It is known (c.f. [1]) that $\bar{i}_{S}^{q}$ can be splitted into injections

$$
i_{S}^{q}: \bar{S}^{q}(E) \stackrel{i_{s}^{q^{\prime}}}{\longrightarrow} S^{1}\left(\bar{S}^{q-1}(E)\right) \stackrel{S^{1}\left(i_{s}^{q-1}\right)}{\longrightarrow} S^{1}\left(\widetilde{S}^{q-1}(E)\right)=\widetilde{S}^{q}(E),
$$

and analogously

$$
i_{T}^{q}: \bar{T}^{q}(E) \stackrel{i_{T}^{q}}{\longrightarrow} T^{1}\left(T^{q-1}(E)\right) \stackrel{T^{1}\left(i_{T^{q-1}}\right)}{\longrightarrow} T^{1}\left(\tilde{T}^{q-1}(E)\right)=\tilde{T}^{q}(E) .
$$

Here the morphism $i_{T}^{q^{\prime}}$ is determined by

$$
\begin{aligned}
\bar{i}_{T}^{q^{\prime}}: e \otimes \sum_{k=0}^{q} \omega_{1}^{k} \otimes \cdots \otimes \omega_{k}^{k} \mapsto & e \otimes \sum_{k=0}^{q-1} \omega_{1}^{k} \otimes \cdots \otimes \omega_{k}^{k} \\
& +e \otimes \sum_{k=0}^{q-1}\left[\omega_{1}^{k+1} \otimes \cdots \otimes \omega_{k}^{k+1}\right] \otimes \omega_{k+1}^{k+1},
\end{aligned}
$$

where $e \in E, \omega_{i}^{k} \in T(M)^{*}$ for $i=1, \cdots k ; k=0, \cdots q ; \omega_{0}^{0}=(1, x) \in \boldsymbol{R}$ and $x \in M$ is the point 'over which' these elements are taken.

One also identifies $E \otimes\left(\bigcirc^{q} T(M)^{*}\right)$ with both the subbundles Ker $\pi_{S}^{q} \subset$ $S^{q}(E)$ as well as $\operatorname{Ker} \pi_{T}^{q} \subset T^{q}(E)$, and $\left.E \otimes^{q} T(M)^{*}\right)$ with both the subbundles $\operatorname{Ker} \bar{\pi}_{S}^{q} \subset \bar{S}^{q}(E)$ as well as $\operatorname{Ker} \bar{\pi}_{T}^{q} \subset \bar{T}^{q}(E)$.

A holonomic or semi-holonomic or non-holonomic pseudo-connection of order $q \geqq 1$ in $E$ is a bundle isomorphism $H H^{q}: S^{q}(E) \rightarrow T^{q}(E)$ or $S H^{q}: \bar{S}^{q}(E) \rightarrow$ $\widetilde{T}^{q}(E)$ or $N H^{q}: \widetilde{S}^{q}(E) \rightarrow \widetilde{T}^{q}(E)$ respectively. Given a sequence $\left\{H H^{q}\right\}_{q=1}^{\infty}$ or $\left\{S H^{q}\right\}_{q=1}^{\infty}$ or $\left\{N H^{q}\right\}_{q=1}^{\infty}$ of pseudo-connections in $E$, then it is called a sequence of holonomic or semi-holonomic or non-holonomic connections if for each $q \geqq 1$, $\pi_{T}^{q} H H^{q}=H H^{q-1} \pi_{S}^{q} ;\left.H H^{q}\right|_{\left.E \otimes(O q T) M)^{*}\right)}=i d$, with $H H^{0}=i d_{E}$, or $\bar{\pi}_{T}^{q} S H^{q}=$ $S H^{q-1} \bar{\pi}_{S}^{q} ;\left.S H^{q}\right|_{E \otimes\left(\otimes^{q} T(M)^{*}\right)}=i d$, with $S H^{0}=i d_{E}$, or $\pi_{T} N H^{q}=N H^{q-1} \pi_{S}$; $\left.N H^{q}\right|_{S^{q-1}(E) \otimes T(M)^{*}}=N H^{q-1} \otimes i d_{T(M)^{*}}$, with $N H^{0}=i d_{E}$.

Remark. These definitions are in accordance with the definitions of higher order connections in vector bundles in [3], [4] or [5]. On the other hand a higher order connection as introduced by C. Ehresmann corresponds in the case of vector bundles to a surconnection (and not connection) of P. Libermann (c.f. [3]). See also [6] for the relation of these two definitions.

As in [1], we restrict our interest to the semi-holonomic and non-holonomic cases. The following sequences of first order pseudo-connections have been also introduced in [1]:

$$
\begin{aligned}
& \left\{\tilde{H}_{S}^{q}\right\}, \text { with } \widetilde{H}_{S}^{q}: S^{1}\left(\widetilde{S}^{q-1}(E)\right) \rightarrow T^{1}\left(\tilde{S}^{q-1}(E)\right) ; \\
& \left\{\tilde{H}_{T}^{q}\right\}, \text { with } \tilde{H}_{T}^{q}: S^{1}\left(\widetilde{T}^{q-1}(E)\right) \rightarrow T^{1}\left(T^{q-1}(E)\right) ;
\end{aligned}
$$




$$
\begin{aligned}
& \left\{\bar{H}_{S}^{q}\right\} \text {, with } \bar{H}_{S}^{q}: S^{1}\left(\bar{S}^{q-1}(E)\right) \rightarrow T^{1}\left(\bar{S}^{q-1}(E)\right) ; \\
& \left\{\bar{H}_{T}^{q}\right\}, \text { with } \bar{H}_{T}^{q}: S^{1}\left(\bar{T}^{q-1}(E)\right) \rightarrow T^{1}\left(\bar{T}^{q-1}(E)\right) .
\end{aligned}
$$

Such a sequence $\left\{\tilde{H}_{S}^{q}\right\}$ (or $\left\{\tilde{H}_{T}^{q}\right\}$ ) is called reducible to a sequence $\left\{\bar{H}_{S}^{q}\right\}$ (or $\left.\left\{\bar{H}_{T}^{q}\right\}\right)$ if for each $q \geqq 1$ the relation $\widetilde{H}_{S}^{q} S^{1}\left(\bar{i}_{S}^{q-1}\right)=T^{1}\left(i_{S}^{q-1}\right) \bar{H}_{S}^{q}\left(\right.$ or $\widetilde{H}_{T}^{q} S^{1}\left(i_{T}^{q-1}\right)=$ $\left.T^{1}\left(i_{T}^{q-1}\right) \bar{H}_{T}^{q}\right)$ holds. A sequence
(a) $\left\{S H^{q}\right\}$;
(b) $\left\{\bar{H}_{S}^{q}\right\}$;
(c) $\left\{\bar{H}_{T}^{q}\right\}$

of pseudo-connections is called regular if for each $q \geqq 1$ the following condition is satisfied:

(a) $\bar{\pi}_{T}^{q} S H^{q}=S H^{q-1} A^{q-1} \bar{\pi}_{S}^{q}$ for some sequence $\left\{A^{q-1}\right\}$ of automorphisms $A^{q-1}: \bar{S}^{q-1}(E) \rightarrow \bar{S}^{q-1}(E)$ or, equivalently, $\bar{\pi}_{S}^{q}\left(S H^{q}\right)^{-1}=\left(S H^{q-1}\right)^{-1}\left(B^{q-1}\right)^{-1}$ $\bar{\pi}_{T}^{q}$ for some sequence $\left\{B^{q-1}\right\}$ of automorphisms $B^{q-1}: \bar{T}^{q-1}(E) \rightarrow \bar{T}^{q-1}(E)$;

(b) $\pi_{T} \bar{H}_{S}^{q} l_{S}^{q^{\prime}}=A^{q-1} \bar{\pi}_{S}^{q}$ and $T^{1}\left(A^{q-1} \bar{\pi}_{S}^{q}\right) \bar{H}_{S}^{q+1} \imath_{S}^{q+1^{\prime}}=\bar{H}_{S}^{q} l_{S}^{q^{\prime}} A^{q} \bar{\pi}_{S}^{q+1}$ for some sequence $\left\{A^{q-1}\right\}$ of automorphisms as sub $(a)$;

(c) $\pi_{S}\left(\bar{H}_{T}^{q}\right)^{-1} i_{T}^{q^{\prime}}=\left(B^{q-1}\right)^{-1} \bar{\pi}_{T}^{q}$ and $S^{1}\left(\left(B^{q-1}\right)^{-1} \bar{\pi}_{T}^{q}\right)\left(\bar{H}_{T}^{q+1}\right)^{-1} \bar{i}_{T}^{q+1^{\prime}}=$ $\left(\bar{H}_{T}^{q}\right)^{-1} \bar{i}_{T}^{q^{q}}\left(B^{q}\right)^{-1} \bar{\pi}_{T}^{q+1}$ for some sequence $\left\{B^{q-1}\right\}$ of automorphisms as sub (a).

The relations

$$
N H^{q}=T^{1}\left(N H^{q-1}\right) \widetilde{H}_{S}^{q} \Longleftrightarrow \widetilde{H}_{S}^{q}=T^{1}\left(N H^{q-1}\right)^{-1} N H^{q}
$$

and

$$
N H^{q}=\tilde{H}_{T}^{q} S^{1}\left(N H^{q-1}\right)\left\langle\Rightarrow \widetilde{H}_{T}^{q}=N H^{q} S^{1}\left(N H^{q-1}\right)^{-1}\right.
$$

define a 'one-to-one-to-one' correspondence $\left\{\widetilde{H}_{S}^{q}\right\} \sim\left\{N H^{q}\right\} \sim\left\{\tilde{H}_{T}^{q}\right\}$ between the three sequences dealt with in the non-holonomic case. The main theorem in [1] states that if there is a triple of sequences in such a correspondence, then the following conditions are equivalent:

(I) $\left\{N H^{q}\right\}$ is reducible to a regular sequence $\left\{S H^{q}\right\}$ with the automorphisms $\left\{A^{q-1}\right\}$ (or $\left\{B^{q-1}=S H^{q-1} A^{q-1}\left(S H^{q-1}\right)^{-1}\right\}$ );

(II) $\left\{\widetilde{H}_{S}^{q}\right\}$ is reducible to a regular sequence $\left\{\bar{H}_{S}^{q}\right\}$ with the automorphisms $\left\{A^{q-1}\right\}$; $\left\{B^{q-1}\right\}$.

(III) $\left\{\tilde{H}_{T}^{q}\right\}$ is reducible to a regular sequence $\left\{\tilde{H}_{T}^{q}\right\}$ with the automorphisms

In particular it has been shown there that if $H$ is a (first order) connection in $E, h$ a (first order) connection in the tangent bindle $T(M)$, then one can get 'by prolongation' sequences which satisfy (III) and hence all the above conditions. This can be generalized with some restrictions to the case where $H$ is a skew connection in $E, h$ a skew connection in $T(M)$.

Thus suppose $H \in S C(E)$ with the tensors $A$ and $Q, h \in S C(T(M))$ with the tensors $a$ and $q$ are $R$-linked skew connections, i.e. $Q=P \otimes R, q=p \otimes R$ for 
some fixed bundle automorphism $R: T(M)^{*} \rightarrow T(M)^{*}$. We have already seen that one can construct then two canonical sequences $\left\{\bar{H}_{T}^{q}\right\}$ and $\left\{\widetilde{H}_{T}^{q}\right\}$, where each $\bar{H}_{T}^{q}(q \geqq 1)$ is a skew connection in $\bar{T}^{q-1}(E)$ with the first tensor $\bar{A}_{T}^{q}=A \otimes \sum_{k=0}^{q}$ $\otimes^{k} a^{*}$, the second tensor $\bar{Q}_{T}^{q}=\bar{P}_{T}^{q} \otimes R$, with $\bar{P}_{T}^{q}=P \otimes \sum_{k=0}^{q} \otimes p^{*}$, and each $\widetilde{H}_{T}^{q}(q \geqq 1)$ is a skew connection in $\widetilde{T}^{q-1}(E)$ with the first tensor $\tilde{A}_{T}^{q}=A \otimes$ $\left(\otimes^{q-1}\left(i d_{R} \otimes a^{*}\right)\right)$, the second tensor $\widetilde{Q}_{T}^{q}=\widetilde{P}_{T}^{q} \otimes R$, with $\widetilde{P}_{T}^{q}=P \otimes\left(\otimes^{q-1}\left(i d_{R} \otimes\right.\right.$ $\left.p^{*}\right)$ ). Denote by $\left\{\left(\bar{H}_{T}^{q}\right)^{0}\right\}$ and $\left\{\left(\tilde{H}_{T}^{q}\right)^{0}\right\}$ the sequences of the corresponding associated connections - they are constructed from the associated to $H$ and $h$ connections $H^{0}$ and $h^{0}$ respectively as in [1].

Lemma 5. The sequence $\left\{\tilde{H}_{T}^{q}\right\}$ is reducible to the sequence $\left\{\bar{H}_{T}^{q}\right\}$, i.e. for each $q \geqq 1$,

$$
H_{\Gamma}^{q} S^{1}\left(i_{T}^{q-1}\right)=T^{1}\left(i_{T}^{q-1}\right) \bar{H}_{T}^{q} .
$$

Proof. Such a relation certainly holds for the sequences $\left\{\left(\tilde{H}_{T}^{q}\right)^{0}\right\}$ and $\left\{\left(\bar{H}_{T}^{q}\right)^{0}\right\}$ (c.f. [1] or [2]). On the other hand the relation between skew connections and associated connections gives in this case

$$
\begin{aligned}
& \tilde{H}_{T}^{q}=j_{T}^{1} \widetilde{A}_{T}^{q} \pi_{T}\left(\widetilde{H}_{T}^{q}\right)^{0}+j_{T}^{1 *}\left(\widetilde{P}_{T}^{q} \otimes R\right) \pi_{T}^{*}\left(\widetilde{H}_{T}^{q}\right)^{0}, \\
& \bar{H}_{T}^{q}=j_{T}^{1} \bar{A}_{T}^{q} \pi_{T}\left(\bar{H}_{T}^{q}\right)^{0}+j_{T}^{1^{*}}\left(\bar{P}_{T}^{q} \otimes R\right) \pi_{T}^{*}\left(\bar{H}_{T}^{q}\right)^{0},
\end{aligned}
$$

and thus by (2.14-15) and (2.67-68) of [1] we get subsequently

$$
\begin{aligned}
\widetilde{H}_{T}^{q} S^{1}\left(\bar{i}_{T}^{q-1}\right) & =j_{T}^{1} \tilde{A}_{T}^{q} \pi_{T} T^{1}\left(\bar{i}_{T}^{q-1}\right)\left(\bar{H}_{T}^{q}\right)^{0}+j_{T}^{1^{*}}\left(\widetilde{P}_{T}^{q} \otimes R\right) \pi_{T}^{*}\left(T^{1}\left(i_{T}^{q-1}\right)\left(\bar{H}_{T}^{q}\right)^{0}\right. \\
& =j_{T}^{1} \tilde{A}_{T}^{q} i_{T}^{q-1} \pi_{T}\left(\bar{H}_{T}^{q}\right)^{0}+j_{T}^{l^{*}}\left(\tilde{P}_{T}^{q} \otimes R\right)\left(i_{T}^{q-1} \otimes i d_{T(M)^{*}}\right) \pi_{T}^{*}\left(\bar{H}_{T}^{q}\right)^{0} \\
& =j_{T}^{1} \bar{i}_{T}^{q-1} \bar{A}_{T}^{q} \pi_{T}\left(\bar{H}_{T}^{q}\right)^{0}+j_{T}^{1^{*}}\left(\bar{i}_{T}^{q-1} \otimes i d_{T(M)^{*}}\right)\left(\bar{P}_{T}^{q} \otimes R\right) \pi_{T}^{*}\left(\bar{H}_{T}^{q}\right)^{0} \\
& =j_{T}^{1} i_{T}^{q-1} \pi_{T} j_{T}^{1} \bar{A}_{T}^{q} \pi_{T}\left(\bar{H}_{T}^{q}\right)^{0}+j_{T}^{1^{*}}\left(\bar{i}_{T}^{q-1} \otimes i d_{T(M)^{*}}\right) \pi_{T}^{*} j_{T}^{1^{*}}\left(\bar{P}_{T}^{q} \otimes R\right) \pi_{T}^{*}\left(\bar{H}_{T}^{q}\right)^{0} \\
& =T^{1}\left(\bar{i}_{T}^{q-1}\right) \tilde{H}_{T}^{q} .
\end{aligned}
$$

Here we have used the obvious relations

$$
A_{T}^{q} i_{T}^{q-1}=i_{T}^{q-1} \bar{A}_{T}^{q} \text { and } \tilde{P}_{T}^{q} \bar{l}_{T}^{q-1}=i_{T}^{q-1} \bar{P}_{T}^{q} .
$$

THEOREM 3. Let $H$ be a skew connection in $E$ with the tensors $A$ and $Q=P \otimes R$ which is regular and such that $A$ commutes with $P$. Let h be a skew connection in $T(M)$ with a trivial first tensor (i.e. $\left.a=i d_{T(M)}\right)$ and the second tensor $q=p \otimes R$ (especially let $h$ be a connection in $T(M))$. Then the canonical sequence $\left\{\tilde{H}_{T}^{q}\right\}$ of skew connections is reducible to the canonical sequence $\left\{\bar{H}_{T}^{q}\right\}$, which is regular.

PRoof. According to Lemma 5, all we have to prove is that $\left\{\bar{H}_{T}^{q}\right\}$ is regular. By the corollary of Lemma 4 we easily conclude, that each skew connection $\bar{H}_{T}^{q}$ is regular, i.e. $\bar{A}_{T}^{q}$-invariant, i.e. $T^{1}\left(\bar{A}_{T}^{q+1}\right) \bar{H}_{T}^{q+1}=\bar{H}_{T}^{q+1} S^{1}\left(\bar{A}_{T}^{q+1}\right) \Rightarrow S^{1}\left(\bar{\pi}_{T}^{q}\right)$ $S^{1}\left(\bar{A}_{T}^{q+1}\right)^{-1}\left(\bar{H}_{T}^{q+1}\right)^{-1} i_{T}^{q+1}=S^{1}\left(\bar{\pi}_{T}^{q}\right)\left(\bar{H}_{T}^{q+1}\right)^{-1} T^{1}\left(\bar{A}_{T}^{q+1}\right)^{-1} \bar{i}_{T}^{q+1}$. Now we have evidently $S^{1}\left(\bar{\pi}_{T}^{q}\right) S^{1}\left(\bar{A}_{T}^{q+1}\right)^{-1}=S^{1}\left(\bar{A}_{T}^{q}\right)^{-1} S^{1}\left(\bar{\pi}_{T}^{q}\right)$, and from (4) we also derive $T^{1}\left(\bar{\pi}_{T}^{q}\right) \bar{H}_{T}^{q+1}=\bar{H}_{T}^{q} S^{1}\left(\bar{\pi}_{T}^{q}\right)$, i.e. $S^{1}\left(\bar{\pi}_{T}^{q}\right)\left(\bar{H}_{T}^{q+1}\right)^{-1}=\left(\tilde{H}_{T}^{q}\right)^{-1} T^{1}\left(\bar{\pi}_{T}^{q}\right)$. Finally by 
(2.64) of [1] we get $T^{1}\left(\bar{\pi}_{T}^{q}\right) T^{1}\left(\bar{A}_{T}^{q+1}\right)^{-1} i_{T}^{p+1^{\prime}}=T^{1}\left(\bar{A}_{T}^{q}\right)^{-1} \bar{i}_{T}^{q^{\prime}} \bar{\pi}_{T}^{q+1}$ and this completes the proof, since we have

$$
T^{1}\left(\bar{A}_{T}^{q}\right)^{-1} \bar{l}_{T}^{q^{\prime}}=\bar{i}_{T}^{q^{\prime}}\left(\bar{A}_{T}^{q+1}\right)^{-1}
$$

because of $a=i d_{T(M)}$.

The results just obtained can be summarized in the following way: If the assumptions of Theorem 3 are satisfied - especially if $H$ is a regular relative connection in $E$ and $h$ a connection in $T(M)$ - then the prolongation procedure described in [1] and [2] 'works' in essentially the same manner as for connections. That is, we get a canonical sequence $\left\{N H^{q}\right\}$ of non-holonomic pseudo-connections in $E$ reducible to a regular sequence $\left\{S H^{q}\right\}$ of semi-holonomic pseudo-connections in $E$, and they are uniquely connected also with a sequence $\left\{\tilde{H}_{S}^{q}\right\}$ of first order pseudo-connections in the nonholonomic jet prolongations of $E$ reducible to a regular sequence $\left\{\bar{H}_{S}^{q}\right\}$ of pseudo-connections in the semi-holonic jet prolongations of $E$. Since $\left\{S H^{q}\right\}$ is regular, $\bar{\pi}_{S}^{q}(X)=0 \Rightarrow \bar{\pi}_{T}^{q} S H^{q}(X)=0$, and we have also

THEOREM 4. Under the assumptions of Theorem 3 , all the $\tilde{H}_{S}^{q}$ and $\bar{H}_{S}^{q}$ are skew connections.

Proof. By (4.8-9) of [1], $\tilde{H}_{S}^{q}=T^{1}\left(N H^{q-1}\right)^{-1} N H^{q}=T_{1}\left(N H^{q-1}\right)^{-1} \widetilde{H}_{T}^{q}$ $S^{1}\left(N H^{q-1}\right)$, i.e. $\pi_{T} \widetilde{H}_{S}^{q}=\left(N H^{q-1}\right)^{-1} \widetilde{A}_{T}^{q} N H^{q-1} \pi_{S}$, which proves that $\widetilde{H}_{S}^{q}$ is a skew connection. Similarly $\pi_{S}(X)=0 \Rightarrow \pi_{S} S^{1}\left(S H^{q-1}\right)(X)=S H^{q-1} \pi_{S}(X)=0$ and thus also $\pi_{T} \bar{H}_{T}^{q} S^{1}\left(S H^{q-1}\right)=0$ which means by (4.44) of [1] that $S H^{q-1}$ $\pi_{T} \bar{H}_{S}^{q}=0$, i.e. $\bar{H}_{S}^{q}$ is a skew connection.

One can define, in an evident manner, the functors $\bar{T}^{q}, \tilde{T}^{q}, \bar{S}^{q}, \tilde{S}^{q}$ from the category of vector bundles over $M$ into itself. Note that for $A: E \rightarrow E$ we have by our notations now $\bar{T}^{q}(A)=\bar{A}_{T}^{q+1}, \tilde{T}^{q}(A)=\tilde{A}_{T}^{q+1}$, and $\tilde{S}^{q}(A)=S^{1}\left(\tilde{S}^{q-1}(A)\right)$ recurrently also satisfies

$$
i_{S}^{q} \bar{S}^{q}(A)=\tilde{S}^{q}(A) \bar{i}_{S}^{q} .
$$

THEOREM 5. If $H$ is a regular A-connection in $E$ and $h$ a connection in $T(M)$ then the canonical prolongations are such that each $\widetilde{H}_{T}^{q}$ is a $\widetilde{T}^{q-1}(A)$-connection, each $\bar{H}_{T}^{q}$ is a $\bar{T}^{q-1}(A)$-connection, each $\tilde{H}_{S}^{q}$ is a $\widetilde{S}^{q-1}(A)$-connection, and each $\bar{H}_{S}^{q}$ is a $\bar{S}^{q-1}(A)$-connection.

Proof. The statement is evident for the $\widetilde{H}_{T}^{q}$ and $\bar{H}_{T}^{q}$ from their construction. We shall first show that for $q \geqq 1$

$$
N H^{q} \tilde{S}^{q}(A)=\tilde{T}^{q}(A) N H^{q} .
$$

This being evident for $q=1$, we proceed by induction using (2) and get $N H^{q} \widetilde{S}^{q}(A)$ $=\tilde{H}_{T}^{q} S^{1}\left(N H^{q-1} \widetilde{S}^{q-1}(A)\right)=\tilde{H}_{T}^{q} S^{1}\left(\tilde{T}^{q-1}(A)\right) S^{1}\left(N H^{q-1}\right)=\widetilde{T}^{q}(A) \tilde{H}_{T}^{q} S^{1}\left(N H^{q-1}\right)$ $=\tilde{T}^{q}(A) N H^{q}$, because by the Corollary of Lemma 4 the skew connection $\tilde{H}_{T}^{q}$ is regular. Using this relation we have as in the proof of the preceding theorem 
$\pi_{T} \widetilde{H}_{S}^{q}=\left(N H^{q-1}\right)^{-1} \widetilde{T}^{q-1}(A) N H^{q-1} \pi_{S}=\widetilde{S}^{q-1}(A) \pi_{S} . \quad$ Also if $X \in \operatorname{Ker} \pi_{S}=$ $\tilde{T}^{q-1}(E) \otimes T(M)^{*} \subset S^{1}\left(\widetilde{T}^{q-1}(E)\right)$ then $\tilde{H}_{T}^{q}(X)=\left(T^{q-1}(A) \otimes i d_{T(M)^{*}}\right)(X)$ and

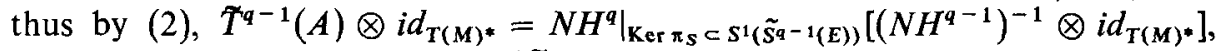
i.e. by $(7),\left.N H^{q}\right|_{\text {Ker } \pi_{S}}=N H^{q-1} \tilde{S}^{q-1}(A) \otimes i d_{T(M)^{*}}$. But then for $X \in \operatorname{Ker} \pi_{S}$ $\subset S^{1}\left(\tilde{S}^{q-1}(E)\right)$ we have again by $(2), \tilde{H}_{S}^{q}(X)=T^{1}\left(N H^{q-1}\right)^{-1} N H^{q}(X)=$ $\left[\left(N H^{q-1}\right)^{-1} \otimes i d_{T(M)^{*}}\right]\left[N H^{q-1} \tilde{S}^{q-1}(A) \otimes i d_{T(M)^{*}}\right](X)$, from where we conclude that $\tilde{H}_{S}^{q}$ is a $\widetilde{S}^{q-1}(A)$-connection, As for the $\bar{H}_{S}^{q}$, consider (6) together with the reducibility condition of $\left\{\tilde{H}_{S}^{q}\right\}$ to $\left\{\bar{H}_{S}^{q}\right\}$. From the just proved result about $\widetilde{H}_{S}^{q}$ we get $i_{S}^{q-1} \pi_{T} \bar{H}_{S}^{q}=\pi_{T} T^{1}\left(\bar{i}_{S}^{q-1}\right) \bar{H}_{S}^{q}=\widetilde{S}^{q-1}(A) \pi_{S} S^{1}\left(i_{S}^{q-1}\right)=\widetilde{S}^{q-1}(A)$ $i_{S}^{q-1} \pi_{S}=i_{S}^{q-1} \bar{S}^{q-1}(A) \pi_{S}$, and hence $\pi_{T} \bar{H}_{S}^{q}=\bar{S}^{q-1}(A) \pi_{S}$, because $i_{S}^{q-1}$ is injective. Also if $X \in \operatorname{Ker} \pi_{S} \subset S^{1}\left(\bar{S}^{q-1}(E)\right)$, then $S^{1}\left(i_{S}^{q-1}\right)(X) \in \operatorname{Ker} \pi_{S} \subset S^{1}\left(\tilde{S}^{q-1}(E)\right)$ and thus by the already proved result about $\tilde{H}_{S}^{q}$ we have $T^{1}\left(i_{S}^{q-1}\right) \bar{H}_{S}^{q}(X)$ $=\tilde{H}_{S}^{q} S^{1}\left(\bar{i}_{S}^{q-1}\right)(X)=\left(\tilde{S}^{q-1}(A) \otimes i d_{T(M)^{*}}\right) S^{1}\left(i_{S}^{q-1}\right)(X)=\left(\tilde{S}^{q-1}(A) i_{S}^{q-1} \otimes i d_{T(M)^{*}}\right)$ $(X)=\left(i_{S}^{q-1} \otimes i d_{T(M)^{*}}\right)\left(\tilde{S}^{q-1}(A) \otimes i d_{T(M)^{*}}\right)(X)=T^{1}\left(\bar{i}_{S}^{q-1}\right)\left(\bar{S}^{q-1}(A) \otimes i d_{T(M)^{*}}\right)(X)$, and this proves the last relation because of the injectivity of $T^{1}\left(i_{S}^{q-1}\right)$.

THEOREM 6. Under the assumptions of Theorem 5, all the relative connections $\tilde{H}_{T}^{q}, \bar{H}_{T}^{q}, \widetilde{H}_{S}^{q}, \bar{H}_{S}^{q}$ are regular.

Proof. It is again evident from the Corollary of Lemma 4 that this is true for $\tilde{H}_{T}^{q}$ and $\bar{H}_{T}^{q}$. Thus we have only to prove $T^{1}\left(\tilde{S}^{q-1}(A)\right) \tilde{H}_{S}^{q}=\tilde{H}_{S}^{q} \widetilde{S}^{q}(A)$, and $T^{1}\left(\bar{S}^{q-1}(A)\right) \bar{H}_{S}^{q}=\bar{H}_{S}^{q} S^{1}\left(\bar{S}^{q-1}(A)\right)$. The first relation follows by (2) and (3) from (7) as $\tilde{H}_{S}^{q} \tilde{S}_{q}(A)=T^{1}\left(N H^{q-1}\right)^{-1} N H^{q} \tilde{S}^{q}(A)=T^{1}\left(\left(N H^{q-1}\right)^{-1} \widetilde{T}^{q-1}(A)\right)$ $N H^{q}=T^{1}\left(\widetilde{S}^{q-1}(A)\right) T^{1}\left(N H^{q-1}\right)^{-1} N H^{q}=T^{1}\left(\widetilde{S}^{q-1}(A)\right) \tilde{H}_{S}^{q}$. The second relation is obtained from this, the reducibility of $\left\{\tilde{H}_{S}^{q}\right\}$ to $\left\{\bar{H}_{S}^{q}\right\}$ and $(6)$ as $T^{1}\left(i_{S}^{q-1}\right) \bar{H}_{S}^{q}$ $S^{1}\left(\bar{S}^{q-1}(A)\right)=\tilde{H}_{S}^{q} S^{1}\left(i_{S}^{q-1} \bar{S}^{q-1}(A)\right)=\tilde{H}_{S}^{q} S^{1}\left(\widetilde{S}^{q-1}(A)\right) S^{1}\left(i_{S}^{q-1}\right)=T^{1}\left(\widetilde{S}^{q-1}(A)\right) \tilde{H}_{S}^{q}$ $S^{1}\left(i_{S}^{q-1}\right)=T^{1}\left(\tilde{S}^{q-1}(A)\right) T^{1}\left(\bar{\imath}_{S}^{q-1}\right) \bar{H}_{S}^{q}=T^{1}\left(\bar{i}_{S}^{q-1}\right) T^{1}\left(\bar{S}^{q-1}(A)\right) \bar{H}_{S}^{q}$, Q.E.D., since $T^{1}\left(i_{S}^{q-1}\right)$ is injective.

REMARK. Restricting ourselves to the most important case of a skew connection, namely to that of a relative connection, we have seen here that 'the prolongation procedure works' only if the initial (regular) relative connection in $E$ is 'pushed' by a (strict) connection in $T(M)$. This is due to our definition of the regularity of the sequence $\left\{\bar{H}_{T}^{q}\right\}$. If $H$ and $h$ were both arbitrary regular relative connections, we would still get the prolonged sequence $\left\{\tilde{H}_{T}^{q}\right\}$ reducible to $\left\{\bar{H}_{T}^{q}\right\}$, however not necessarily regular, the obstacle being essentially only with the relation (5). It seems likely that one could generalize the notion of a relative connection (most probably by developing the formalism in the category of 'all' vector bundles rather than only of those over a fixed $M$ ), and get a deeper condition for the 'initial' correlation (of the relative connection in $T(M)$ to the relative connection in $E$ ) in order to 'let the prolongation procedure work'. 


\section{References}

[1] J. Virsik, Non-holonomic connections on vector bundles, Czech. Math. J. 17 (1967), I: 108-147; II: 200-224.

[2] J. Virsik, Ưber Zusammenhänge höherer Ordnungen in Vektorraumbündeln, Math. Nachrichten 34 (1967), 219-227.

[3] E. Feldmann, The geometry of immersions, Bull. Amer. Math. Soc. 69 (1963), 693-698.

[4] P. Libermann, Sur la géométrie des prolongements des espaces fibrés vectoriels, Ann. Inst. Fourier, Grenoble 14 (1964), 145-172.

[5] W. Pohl, Connections in differential geometry of higher order, Transactions Amer. Math. Soc. 125 (1966), 310-325.

[6] N. Que, Du prolongement des espaces fibrés et des structures infinitésimales, Ann. Inst. Fourier, Grenoble 17 (1967), 157-223.

\section{Monash University}

